\title{
Symmetric Functions and Generating Functions for Descents and Major Indices in Compositions
}

\author{
Evan Fuller ${ }^{1}$ and Jeffrey Remmel ${ }^{2}$ \\ ${ }^{1}$ Department of Mathematics, Montclair State University, Montclair, New Jersey 07043, USA \\ fullere@montclair.edu \\ ${ }^{2}$ Department of Mathematics, University of California, San Diego, La Jolla, CA 92093-0112, \\ USA \\ remmel@math.ucsd.edu
}

Received November 18, 2008

AMS Subject Classification: 05A15, 05E05

\begin{abstract}
In [18], Mendes and Remmel showed how Gessel's generating function for the distributions of the number of descents, the major index, and the number of inversions of permutations in the symmetric group could be derived by applying a ring homomorphism defined on the ring of symmetric functions to a simple symmetric function identity. We show how similar methods may be used to prove analogues of that generating function for compositions.
\end{abstract}

Keywords: permutation statistics, compositions, symmetric functions

\section{Introduction}

A permutation statistic is a function mapping permutations to nonnegative integers. The modern analysis of such objects began in the early twentieth century with the work of MacMahon [16]. He popularized the "classic" notions of the descents, rises, inversions, coinversions, major index, and comajor index statistics. Here if $\sigma=\sigma_{1} \cdots \sigma_{n}$ is an element of the symmetric group $S_{n}$ written in one line notation, then

$$
\begin{gathered}
\operatorname{des}(\sigma)=\sum_{i=1}^{n-1} \chi\left(\sigma_{i}>\sigma_{i+1}\right), \\
\operatorname{ris}=1+\sum_{i=1}^{n-1} \chi\left(\sigma_{i}<\sigma_{i+1}\right), \operatorname{inv}(\sigma)=\sum_{1 \leq i<j \leq n} \chi\left(\sigma_{i}>\sigma_{j}\right), \operatorname{coinv}(\sigma)=\sum_{1 \leq i<j \leq n} \chi\left(\sigma_{i}<\sigma_{j}\right), \\
\operatorname{maj}(\sigma)=\sum_{i=1}^{n-1} i \chi\left(\sigma_{i}>\sigma_{i+1}\right), \quad \operatorname{comaj}(\sigma)=\sum_{i=1}^{n-1} i \chi\left(\sigma_{i}<\sigma_{i+1}\right),
\end{gathered}
$$

where for any statement $A, \chi(A)$ is 1 if $A$ is true and 0 if $A$ is false. These definitions make sense if $\sigma=\sigma_{1} \cdots \sigma_{n}$ is any sequence of natural numbers. 
The study of the properties of these statistics and subsequent generalizations of these statistics to other groups and sequences remains an active area of research today. In this paper, we shall find analogues of the joint distribution of $\operatorname{des}(\sigma), \operatorname{maj}(\sigma)$, and $\operatorname{inv}(\sigma)$. That is, Gessel gave a generating function for

$$
\sum_{\sigma \in S_{n}} x^{\operatorname{des}(\sigma)} u^{\operatorname{maj}(\sigma)} q^{\operatorname{inv}(\sigma)},
$$

both in his thesis and in a paper coauthored with Garsia [9, 10]. Later, Mendes and Remmel showed how Gessel's result could be derived by applying a homomorphism defined on the ring of symmetric functions $\Lambda$ in infinitely many variables $x_{1}, x_{2}, \ldots$ to the simple symmetric function identity

$$
H(t)=\frac{1}{E(-t)},
$$

where $H(t)$ is the generating function for the homogeneous symmetric functions $h_{n}=h_{n}\left(x_{1}, x_{2}, \ldots\right)$ and $E(t)$ is the generating function for the elementary symmetric functions $e_{n}=e_{n}\left(x_{1}, x_{2}, \ldots\right)$. That is,

$$
H(t)=\sum_{n \geq 0} h_{n} t^{n}=\prod_{n \geq 1} \frac{1}{1-x_{i} t}
$$

and

$$
E(t)=\sum_{n \geq 0} e_{n} t^{n}=\prod_{n \geq 1}\left(1+x_{i} t\right) .
$$

In particular, Mendes and Remmel proved the following formula, which is easily derived from the Garsia-Gessel formula for the generating function of $\operatorname{des}(\sigma), \operatorname{maj}(\sigma)$, and $\operatorname{inv}(\sigma)$,

$$
\begin{aligned}
& \sum_{n \geq 0} \frac{t^{n}}{[n]_{p, q} !(x, y ; u, v)_{n+1}} \sum_{\sigma \in S_{n}} x^{\operatorname{des}(\sigma)} y^{\operatorname{ris}(\sigma)} u^{\operatorname{maj}(\sigma)} v^{\operatorname{comaj}(\sigma)} q^{\operatorname{inv}(\sigma)} p^{\operatorname{coinv}(\sigma)} \\
& =\sum_{k \geq 0} \frac{x^{k}}{y^{k+1} \mathbf{e}_{p, q}^{-t(u / v)^{0}} \ldots \mathbf{e}_{p, q}^{-t(u / v)^{k}}} .
\end{aligned}
$$

Here we use standard notation from hypergeometric function theory. For $n \geq 1$ and $\lambda \vdash n$, let

$$
[n]_{p, q}=\frac{p^{n}-q^{n}}{p-q}=p^{n-1} q^{0}+\cdots+p^{0} q^{n-1}
$$

and

$$
[n]_{p, q} !=[n]_{p, q} \cdots[1]_{p, q}
$$

be the $p, q$-analogues of $n$ and $n !$. By convention, let $[0]_{p, q}=0$ and $[0]_{p, q} !=1$. We let $(x ; q)_{0}=1$ and

$$
(x ; q)_{n}=(1-x)(1-x q) \cdots\left(1-x q^{n-1}\right) .
$$


In addition, let $(x, y ; p, q)_{0}=1$ and

$$
(x, y ; p, q)_{n}=(x-y)(x p-y q) \cdots\left(x p^{n-1}-y q^{n-1}\right) .
$$

Finally, $\mathbf{e}_{p, q}^{t}$ is a $p, q$-analog for the exponential function defined by

$$
\mathbf{e}_{p, q}^{t}=\sum_{n \geq 0} \frac{t^{n}}{[n]_{p, q} !} q^{\left(\begin{array}{c}
n \\
2
\end{array}\right)}
$$

Mendes and Remmel also showed how their methods can be used to extend such results to the hyperoctahedral group $B_{n}$ and its subgroup $D_{n}$.

The main goal of this paper is to show how the methods of Mendes and Remmel can prove similar results for compositions. Here a composition $\gamma$ is a sequence of positive integers $\gamma=\left(\gamma_{1}, \ldots, \gamma_{k}\right)$. We call the $\gamma_{i}$ 's the parts of $\gamma$ and let $\ell(\gamma)$ denote the number of parts of $\gamma$. We let $|\gamma|=\gamma_{1}+\cdots+\gamma_{k}$ and $x^{\gamma}$ be the monomial $x_{\gamma_{1}} \cdots x_{\gamma_{k}}$. Since compositions can have repeated entries, it is natural to have analogues of des and maj where we replace $>$ by $\geq$ or $=$ in the definition of des and maj. That is, if $\gamma=\left(\gamma_{1}, \ldots, \gamma_{n}\right)$ is a composition, then we let

$$
\begin{aligned}
\operatorname{Des}(\gamma) & =\left\{i: \gamma_{i}>\gamma_{i+1}\right\}, \\
W \operatorname{Des}(\gamma) & =\left\{i: \gamma_{i} \geq \gamma_{i+1}\right\}, \quad \text { and } \\
\operatorname{Lev}(\gamma) & =\left\{i: \gamma_{i}=\gamma_{i+1}\right\} .
\end{aligned}
$$

Then we define

$$
\begin{aligned}
\operatorname{des}(\gamma) & =|\operatorname{Des}(\gamma)|, \\
\operatorname{wdes}(\gamma) & =|\operatorname{DDes}(\gamma)|, \quad \text { and } \\
\operatorname{lev}(\gamma) & =|\operatorname{Lev}(\gamma)|
\end{aligned}
$$

and

$$
\begin{aligned}
\operatorname{maj}(\gamma) & =\sum_{i \in \operatorname{Des}(\gamma)} i, \\
\operatorname{wmaj}(\gamma) & =\sum_{i \in \operatorname{WDes}(\gamma)} i, \quad \text { and } \\
\operatorname{levmaj}(\gamma) & =\sum_{i \in \operatorname{Lev}(\gamma)} i .
\end{aligned}
$$

Let $\mathbb{P}$ denote the set of positive integers. We shall prove the following three theorems.

\section{Theorem 1.1.}

$$
\sum_{n \geq 0} \frac{t^{n}}{(y ; u)_{n+1}} \sum_{\gamma \in \mathbb{P}^{n}} x^{\gamma} y^{\operatorname{des}(\gamma)} u^{\operatorname{maj}(\gamma)}=\sum_{k \geq 0} \frac{y^{k}}{\prod_{i \geq 1}\left(x_{i} t ; u\right)_{k+1}} .
$$




\section{Theorem 1.2.}

$$
\sum_{n \geq 0} \frac{t^{n}}{(y ; u)_{n+1}} \sum_{\gamma \in \mathbb{P}^{n}} x^{\gamma} y^{\operatorname{wdes}(\gamma)} u^{\operatorname{wmaj}(\gamma)}=\sum_{k \geq 0} y^{k} \prod_{i \geq 1}\left(-x_{i} t ; u\right)_{k+1} .
$$

\section{Theorem 1.3.}

$$
\sum_{n \geq 0} \frac{t^{n}}{(y ; u)_{n+1}} \sum_{\gamma \in \mathbb{P}^{n}} x^{\gamma} y^{\operatorname{lev}(\gamma)} u^{\operatorname{levmaj}(\gamma)}=\sum_{k \geq 0} \frac{y^{k}}{\prod_{j=0}^{k}\left(\sum_{n \geq 0} p_{n}\left(-u^{j} t\right)^{n}\right)},
$$

where $p_{n}=p_{n}\left(x_{1}, x_{2}, \ldots\right)=\sum_{i \geq 1} x_{i}^{n}$ is the power symmetric function.

It should be noted that there has been considerable work on enumerating compositions by the number of occurrences of certain patterns in a compositions. For example, if $\gamma=\left(\gamma_{1}, \ldots, \gamma_{k}\right)$ is a composition and we define $\operatorname{ris}(\gamma)=\left|\left\{s: \gamma_{s}<\gamma_{s+1}\right\}\right|$, then Carlitz [7] proved that

$$
\sum_{\gamma \in \mathbb{P}^{*}} u^{\ell(\gamma)} q^{|\gamma|} x^{\mathrm{ris}(\gamma)} y^{\operatorname{des}(\gamma)} z^{\operatorname{lev}(\gamma)}=\frac{e(q u(z-y), q)-e(q u(z-x), q)}{x e(q u(z-x), q)-y e(q u(z-y), q)},
$$

where

$$
e(x, q)=\sum_{n=0}^{\infty} \frac{x^{n}}{(q)_{n}}=\prod_{n=0}^{\infty} \frac{1}{1-q^{n} x}
$$

and $(q)_{0}=1$ and $(q)_{n}=(1-q)\left(1-q^{2}\right) \cdots\left(1-q^{n}\right)$ for $n \geq 1$. Similarly, Heubach and Mansour [13] found generating functions compositions according to the number of occurrences of various 3 letter patterns, and Mansour and Sirhan [17] extended the work of Heubach and Mansour by finding generating functions compositions according to the number of occurrences of various $l$ letter patterns. Enumerating various types of compositions according to other types of patterns can be found in $[12,14]$, and [15]. In each case, one can find such generating functions by applying the transfer matrix method, see [20, Section 4.7] or [11]. The basic idea is the following. Suppose you want to find the generating function

$$
C(u, v, x)=\sum_{\gamma \in \mathbb{P}^{*}} u^{\ell(\gamma)} v^{|\gamma|} x^{\operatorname{des}(\gamma)}
$$

Then one can define

$$
C(i ; u, v, x)=\sum_{\gamma \in \mathbb{P}^{+}, \gamma_{1}=i} u^{\ell(\gamma)} v^{|\gamma|} x^{\operatorname{des}(\gamma)},
$$

and we have simple recursions

$$
C(i ; u, v, x)=u v^{i}+u v^{i}\left(\sum_{j<i} x C(j ; u, v, x)+\sum_{j \geq i} C(j ; u, v, x)\right)
$$

for all $i \geq 1$. Thus, if $U$ and $V$ are the infinite vectors $U=\left[u v^{1}, u v^{2}, \ldots\right]$ and $V=$ $[C(1 ; u, v, x), C(2 ; u, v, x), \ldots]$, we can write down an invertible matrix $M$ such that

$$
U^{T}=M V^{T}
$$


and hence, we can solve for $V^{T}$ as

$$
V^{T}=M^{-1} U^{T}
$$

Then, at least in some cases, one can simplify the expression for $1+\sum_{i>1} C(i ; u, v, x)$ to obtain nice formulas for the desired generating function. Of course, this method is more straightforward if we restrict ourselves to finite alphabets, but it can still work over infinite alphabets, as Carlitz basically showed in [7]. However, when we try the same thing while adding a variable $q$ recording the major index, we cannot derive such an equation. That is, define

$$
C(i ; u, v, x, q)=\sum_{\gamma \in \mathbb{P}^{+}, \gamma_{1}=i} u^{\ell(\gamma)} v^{|\gamma|} x^{\operatorname{des}(\gamma)} q^{\operatorname{maj}(\gamma)} .
$$

When we consider a composition $\gamma=\left(\gamma_{1}, \ldots, \gamma_{k}\right)$ where $\gamma_{1}=j$ and add $i$ to the front of $\gamma$ to obtain the composition $\delta=\left(i, \gamma_{1}, \ldots, \gamma_{k}\right)$, then a descent $\gamma_{s} \geq \gamma_{s+1}$ which contributes $s$ to $\operatorname{maj}(\gamma)$ will contribute $1+s$ to $\operatorname{maj}(\delta)$ since that descent will occur at position $s+1$ in $\delta$. Thus $\operatorname{maj}(\delta)=1+\operatorname{des}(\gamma)+\operatorname{maj}(\gamma)$ if $j<i$ and $\operatorname{maj}(\delta)=$ $\operatorname{des}(\gamma)+\operatorname{maj}(\gamma)$ if $i \leq j$. Hence, in this case we obtain the recursion

$$
C(i ; u, v, x, q)=u v^{i}+u v^{i}\left(\sum_{j<i} q x C(j ; u, v, q x, q)+\sum_{j \geq i} C(j ; u, v, q x, q)\right) .
$$

The fact that $C(j ; u, v, q x, q)$ appears on the right hand side of (1.9) as opposed to the $C(j ; u, v, x, q)$ which appear on the right hand side of (1.7) means that we cannot solve directly for $V^{T}$ in this case. Instead, if $V=V(u, v, x, q)=(C(1 ; u, v, q x, q)$, $C(2 ; u, v, q x, q), C(3 ; u, v, q x, q), \ldots)$ and $A=\left(u v, u v^{2}, u v^{3}, \ldots\right)$, then we end up with an equation of the form

$$
V(u, v, x, q)^{T}=A^{T}+B(u, v, x, q) V(u, v, x q, q)^{T},
$$

where $B(u, v, x, q)$ is a matrix. We can iterate (1.10) to obtain an expression for $V(u, v, x, q)^{T}$ of the form

$$
\begin{aligned}
& A^{T}+B(u, v, x, q) A^{T}+B(u, v, x, q) B(u, v, x q, q) A^{T} \\
& +B(u, v, x, q) B(u, v, x q, q) B\left(u, v, x q^{2}, q\right) A^{T}+\cdots .
\end{aligned}
$$

However, in this case, even when we restrict ourselves to finite alphabets $\{1, \ldots, n\}$ so that the matrix $B(u, v, x, q)$ is finite, this leads to a complicated expression for $V(u, v, x, q)^{T}$. We were unable to see how we could simplify these expressions for $V(u, v, x, q)^{T}$ or $1+\sum_{i=1} C(i ; u, v, x, q)$ to obtain anything as simple as the formula in Theorem 1.1.

It should be noted, however, that various specializations can easily follow from Theorems $1.1,1.2$, and 1.3. That is, by setting the variables $x_{i}=0$ for certain $i$, we can obtain formulas for an arbitrary alphabet $A \subseteq \mathbb{P}$. By setting $x_{i}=u v^{i}$ for all $i$, we can also obtain generating functions like

$$
C(u, v, x, q)=\sum_{\gamma \in \mathbb{P}^{*}} u^{\ell(\gamma)} v^{|\gamma|} x^{\operatorname{des}(\gamma)} q^{\operatorname{maj}(\gamma)}
$$


The outline of this paper is as follows. In Section 2, we will provide the necessary background on symmetric functions and generating functions for partitions that we will need to prove Theorems 1.1, 1.2, and 1.3. In Section 3, we will give the proofs of Theorems 1.1, 1.2, and 1.3, as well as some extensions.

\section{Preliminaries}

To prove Theorems 1.1, 1.2, and 1.3, we will manipulate basic relationships between the elementary and homogeneous symmetric functions. The idea of extracting information about permutation statistics through symmetric function theory has been used for decades, but the methods of this paper - defining a homomorphism on the elementary symmetric functions and evaluating it on the homogeneous symmetric functions - was first given by Brenti [5,6]. Beck and the second author reproved his results combinatorially [2-4]. It is this approach which is closest to our own.

In this section, we shall state the basic results on symmetric functions and generating functions for partitions that we shall need.

We shall consider the ring of symmetric functions $\Lambda$ over infinitely many variables. We let $h_{0}=e_{0}=p_{0}=1$ and for any $n \geq 1$,

$$
\begin{aligned}
& h_{n}=h_{n}\left(x_{1}, x_{2}, \ldots\right)=\sum_{1 \leq i_{1} \leq i_{2} \leq \cdots \leq i_{n}} x_{i_{1}} x_{i_{2}} \cdots x_{i_{n}}, \\
& e_{n}=e_{n}\left(x_{1}, x_{2}, \ldots\right)=\sum_{1 \leq i_{1}<i_{2}<\cdots<i_{n}} x_{i_{1}} x_{i_{2}} \cdots x_{i_{n}}, \text { and } \\
& p_{n}=p_{n}\left(x_{1}, x_{2}, \ldots\right)=\sum_{i \geq 1} x_{i}^{n} .
\end{aligned}
$$

A partition of $n$, written $\lambda \vdash n$, is an increasing sequence of positive integers $\lambda=$ $\left(\lambda_{1}, \lambda_{2}, \ldots, \lambda_{\ell}\right)$ such that $n=\sum_{i=1}^{\ell} \lambda_{i}, \lambda_{1} \leq \lambda_{2} \leq \cdots \leq \lambda_{\ell}$. In such a situation, we write $|\lambda|=n$ and $\ell(\lambda)=\ell$. For any partition $\lambda=\left(\lambda_{1}, \ldots, \lambda_{\ell}\right)$, we define

$$
\begin{aligned}
& h_{\lambda}=h_{\lambda_{1}} \cdots h_{\lambda_{\ell}}, \\
& e_{\lambda}=e_{\lambda_{1}} \cdots e_{\lambda_{\ell}}, \text { and } \\
& p_{\lambda}=p_{\lambda_{1}} \cdots p_{\lambda_{\ell}} .
\end{aligned}
$$

It is well known that the collections $\left\{h_{\lambda}\right\},\left\{e_{\lambda}\right\}$, and $\left\{p_{\lambda}\right\}$ as $\lambda$ runs over all partitions are bases of $\Lambda$. These are referred to as the homogeneous symmetric function basis, the elementary symmetric function basis, and the power symmetric function basis, respectively. These definitions of the homogeneous and elementary symmetric functions give

$$
\sum_{n \geq 0} h_{n} t^{n}=\prod_{i} \frac{1}{1-x_{i} t}=\left(\prod_{i}\left(1-x_{i} t\right)\right)^{-1}=\left(\sum_{n \geq 0} e_{n}(-t)^{n}\right)^{-1} .
$$

This trivial restatement of definitions is the key symmetric function identity behind Theorems 1.1, 1.2, and 1.3. 
A second key ingredient of our proofs is the combinatorial definition of the expansion of $h_{n}$ in terms of the elementary symmetric function basis. This was given by Eğecioğlu and Remmel [8]. A rectangle of height 1 and length $n$ chopped into "bricks" of lengths found in the partition $\lambda$ is known as a brick tabloid of shape $(n)$ and type $\lambda$. For example, Figure 1 shows one brick tabloid of shape (12) and type $(2,3,7)$.

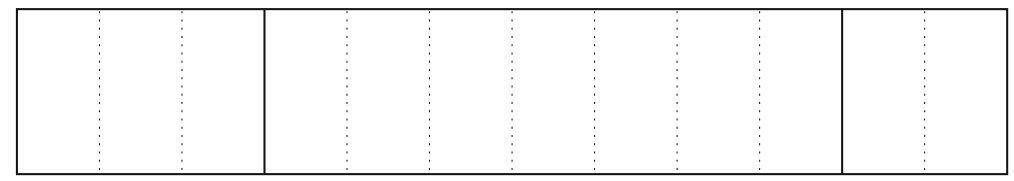

Figure 1: A brick tabloid of shape (12) and type $(2,3,7)$.

Let $B_{\lambda, n}$ be the number of such objects. Through simple recursions, Eğecioğlu and Remmel proved that

$$
h_{n}=\sum_{\lambda \vdash n}(-1)^{n-\ell(\lambda)} B_{\lambda, n} e_{\lambda}
$$

Next, we state several well-known generating functions for partitions, see [1]:

$$
1+\sum_{n \geq 1} \sum_{\lambda \vdash n} q^{|\lambda|} t^{\ell(\lambda)}=\prod_{i \geq 1} \frac{1}{1-t q^{i}}
$$

and

$$
1+\sum_{n \geq 1} \sum_{\substack{\lambda \vdash n, \lambda \text { has distinct parts }}} q^{|\lambda|} t^{\ell(\lambda)}=\prod_{i \geq 1}\left(1+t q^{i}\right) .
$$

More generally, for any set $S \subseteq \mathbb{P}$, let $\operatorname{Ptn}_{n}(S)$ denote the set of partitions of $n$ with parts from $S$. Then,

$$
1+\sum_{n \geq 1} \sum_{\lambda \in P t n_{n}(S)} q^{|\lambda|} t^{\ell(\lambda)}=\prod_{i \in S} \frac{1}{1-t q^{i}}
$$

and

$$
1+\sum_{n \geq 1} \sum_{\substack{\lambda \in \operatorname{Ptn}_{n}(S), \lambda \text { has distinct parts }}} q^{|\lambda|} t^{\ell(\lambda)}=\prod_{i \in S}\left(1+t q^{i}\right)
$$

\section{The Generating Functions for Compositions}

In this section, we shall give our proofs of Theorems 1.1, 1.2, and 1.3.

To prove Theorem 1.1, define a ring homomorphism $\Theta^{(k)}$ by defining it on the elementary symmetric function $e_{n}$ so that

$$
\Theta^{(k)}\left(e_{n}\right)=\left.\sum_{\substack{i_{0}, \ldots, i_{k} \geq 0 \\ i_{0}+\cdots+i_{k}=n}} u^{0 i_{0}+\cdots+k i_{k}}\left[\prod_{j=0}^{k} \prod_{i \geq 1}\left(1+x_{i} z_{j}\right)\right]\right|_{\substack{i_{i} \ldots i_{k} \\ z_{0} \cdots z_{k}^{k}}},
$$


where expression $\left.\right|_{t^{k}}$ means to take the coefficient of $t^{k}$ in expression.

First we apply $\Theta^{(k)}$ to $h_{n}$. We have

$$
\begin{aligned}
\Theta^{(k)}\left(h_{n}\right) & =\sum_{\lambda \vdash n}(-1)^{n-\ell(\lambda)} B_{\lambda, n} \Theta^{(k)}\left(e_{\lambda}\right) \\
& =\left.\sum_{\lambda \vdash n}(-1)^{n-\ell(\lambda)} B_{\lambda, n} \prod_{m=1}^{\ell(\lambda)} \sum_{\substack{i_{0}, \ldots, i_{k} \geq 0 \\
i_{0}+\cdots+i_{k}=\lambda_{m}}} u^{0 i_{0}+\cdots+k i_{k}}\left[\prod_{j=0}^{k} \prod_{i \geq 1}\left(1+x_{i} z_{j}\right)\right]\right|_{z_{0}^{i_{0} \ldots z_{k}}} .
\end{aligned}
$$

Our goal is to interpret $\Theta^{(k)}\left(h_{n}\right)$ as a sum of weighted combinatorial objects. We interpret the sum $\sum_{\lambda \vdash n} B_{\lambda, n}$ as all ways of picking a brick tabloid $T$ of shape $(n)$. Then the factor $(-1)^{n-\ell(\lambda)}$ allows us to place a -1 in each non-terminal cell of a brick in $T$ and place a 1 at the terminal cell of each brick in $T$. Next, for each brick in $T$, choose nonnegative integers $i_{0}, \ldots, i_{k}$ that sum to the total length of the brick. This accounts for the product and second sum in (3.1). Using powers of $u$, these choices for $i_{0}, \ldots, i_{k}$ can be recorded in $T$. In each brick, place a power of $u$ in each cell such that the powers weakly increase from left to right and the number of occurrences of $u^{j}$ is $i_{j}$. At this point, we have constructed an object which may look something like Figure 2 below.

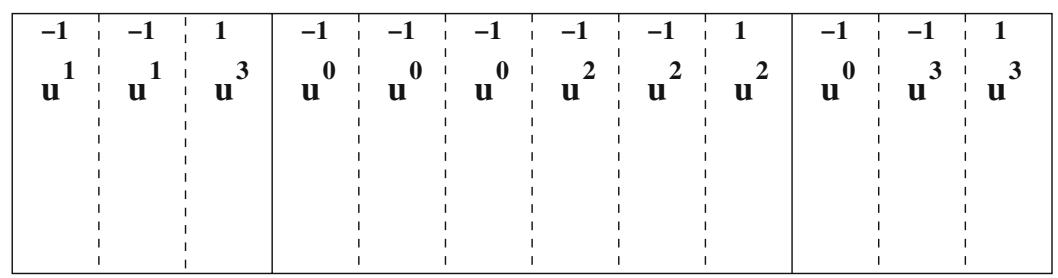

Figure 2: One possible object when $k=3$ and $n=12$.

Now, the term $\left.\left[\prod_{j=0}^{k} \prod_{i \geq 1}\left(1+x_{i} z_{j}\right)\right]\right|_{z_{0}^{i_{0} \ldots z_{k}}}$ let us choose $k+1$ partitions with distinct parts, $\pi^{(0)}, \ldots, \pi^{(k)}$ where $\ell\left(\pi^{(j)}\right)=i_{j}$ for $j=0, \ldots, k$, which we write in strictly decreasing order. Each $i$ that occurs in such a configuration is weighted with $x_{i}$, so that we write these factors in the bottom row of each configuration. Figure 3 gives one example of such an object created in this manner. The weight of such a composite object is the product of the signs at the top of the configuration times the product of the $x_{i}$ 's that appear in the bottom row of the configuration times the products of the $u^{j}$ 's in the second row of the configuration. Thus, the weight of the object in Figure 3 is $-x_{1} x_{2}^{3} x_{3}^{4} x_{4}^{2} x_{5} x_{6} u^{17}$.

These decorated brick tabloids of shape $(n)$ and type $\lambda$ for some $\lambda \vdash n$ have the following properties:

(1) the cells in each brick contain -1 except for the final cell, which contains 1 ,

(2) each cell contains a power of $u$ such that the powers weakly increase within each brick and the largest possible power of $u$ is $u^{k}$, and 


\begin{tabular}{|c|c|c|c|c|c|c|c|c|c|c|c|}
\hline $\begin{array}{r}-1 \\
u^{1}\end{array}$ & $\begin{array}{r}-1 \\
\mathrm{u}^{1}\end{array}$ & $u^{1}$ & $\mathrm{u}^{-1}$ & $\mathbf{u}^{-1}$ & $\begin{array}{c}-1 \\
\mathbf{u}^{0}\end{array}$ & $\mathbf{u}^{-1}$ & $\begin{array}{c}-1 \\
u^{2}\end{array}$ & $\begin{array}{c}\mathbf{1} \\
\mathbf{u}^{2}\end{array}$ & $\begin{array}{c}-1 \\
u^{0}\end{array}$ & $\mathbf{u}^{-1}$ & $\mathbf{u}^{\mathbf{1}}$ \\
\hline 6 & 1 & 3 & 5 & 3 & 2 & 4 & 3 & 2 & 2 & 4 & 3 \\
\hline $\mathbf{x}_{6}$ & $\mathbf{x}$ & $\mathbf{x}_{3}$ & & $\mathbf{x}_{3}$ & & $\mathbf{x}_{4}$ & $\mathbf{x}_{3}$ & $\mathbf{x}_{2}$ & $\mathbf{x}_{2}$ & $\mathbf{x}_{4}$ & $\mathbf{x}_{3}$ \\
\hline
\end{tabular}

Figure 3: An object coming from (3.1) when $k=3$ and $n=12$.

(3) $T$ contains a composition of $n$ which must strictly decrease between consecutive cells within a brick if the cells are marked with the same power of $u$.

In addition, each entry $i$ in the composition is weighted by $x_{i}$. In this way, $\Theta^{(k)}\left(h_{n}\right)$ is the weighted sum over all possible decorated brick tabloids of shape $(n)$.

Next, we define a sign-reversing involution $I$ which will allow us to cancel all the terms $T$ with a negative weight. To define $I$, scan the cells from left to right looking for either a cell containing -1 or two consecutive bricks which may be combined to preserve the properties of this collection of objects. If a -1 is scanned first, break the brick containing the -1 into two immediately after the violation and change the -1 to 1 . If the second situation is scanned first, glue the brick together and change the 1 in the first brick to -1 . For example, the image of Figure 3 is displayed in Figure 4.

\begin{tabular}{|c|c:c|c|c|c|c:c:c|c:c:c|}
\hline $\mathbf{1}$ & $\mathbf{- 1}$ & $\mathbf{1}$ & $\mathbf{- 1}$ & $\mathbf{- 1}$ & $\mathbf{- 1}$ & $\mathbf{- 1}$ & $\mathbf{- 1}$ & $\mathbf{1}$ & $\mathbf{- 1}$ & $\mathbf{- 1}$ & $\mathbf{1}$ \\
$\mathbf{u}$ & $\mathbf{1}$ & $\mathbf{3}$ & $\mathbf{0}$ & $\mathbf{0}$ & $\mathbf{0}$ & $\mathbf{2}$ & $\mathbf{2}$ & $\mathbf{2}$ & $\mathbf{0}$ & $\mathbf{3}$ & $\mathbf{3}$ \\
$\mathbf{6}$ & $\mathbf{1}$ & $\mathbf{3}$ & $\mathbf{5}$ & $\mathbf{3}$ & $\mathbf{2}$ & $\mathbf{4}$ & $\mathbf{3}$ & $\mathbf{2}$ & $\mathbf{2}$ & $\mathbf{4}$ & $\mathbf{3}$ \\
$\mathbf{x}_{\mathbf{6}}$ & $\mathbf{x}_{\mathbf{1}}$ & $\mathbf{x}_{\mathbf{3}}$ & $\mathbf{x}_{\mathbf{5}}$ & $\mathbf{x}_{\mathbf{3}}$ & $\mathbf{x}_{\mathbf{2}}$ & $\mathbf{x}_{\mathbf{4}}$ & $\mathbf{x}_{\mathbf{3}}$ & $\mathbf{x}_{\mathbf{2}}$ & $\mathbf{x}_{\mathbf{2}}$ & $\mathbf{x}_{\mathbf{4}}$ & $\mathbf{x}_{\mathbf{3}}$ \\
\hline
\end{tabular}

Figure 4: The image under $I$ of Figure 3.

It is easy to see that $I$ is a sign-reversing, weight-preserving involution. Thus, $I$ shows that $\Theta^{(k)}\left(h_{n}\right)$ is equal to the sum of the weights of all the fixed points of $I$.

Let's consider the fixed points of $I$. First, there can be no -1 's, so every brick must be of size 1 . Next, it cannot be the case that the power of $u$ strictly increases as we move from brick $i$ to brick $i+1$, since then we could combine these two bricks and still satisfy properties (1), (2), and (3). Thus, the powers of $u$ must weakly decrease as we read from left to right. Let $\gamma=\left(\gamma_{1}, \ldots, \gamma_{n}\right)$ denote the underlying composition. We note that if the power of $u$ is the same on brick $i$ and $i+1$, then it must be the case that $\gamma_{i} \leq \gamma_{i+1}$; otherwise, we could combine brick $i$ and brick $i+1$. One example of a fixed point may be found in Figure 5.

We now turn our attention to counting fixed points.

Suppose that the powers of $u$ in a fixed point are $r_{1}, \ldots, r_{n}$ when read from left to right. It must be the case that $k \geq r_{1} \geq \cdots \geq r_{n}$. Define nonnegative integers $a_{i}$ by $a_{i}=r_{i}-r_{i+1}$ for $i=1, \ldots, n-1$ and let $a_{n}=r_{n}$. It follows that $r_{1}+\cdots+r_{n}=$ 


\begin{tabular}{|c|c|c|c|c|c|c|c|c|c|c|c|}
\hline $\mathbf{1}$ & $\mathbf{1}$ & $\mathbf{1}$ & $\mathbf{1}$ & $\mathbf{1}$ & $\mathbf{1}$ & $\mathbf{1}$ & $\mathbf{1}$ & $\mathbf{1}$ & $\mathbf{1}$ & $\mathbf{1}$ & $\mathbf{1}$ \\
$\mathbf{u}^{3}$ & $\mathbf{u}^{3}$ & $\mathbf{u}^{3}$ & $\mathbf{u}^{3}$ & $\mathbf{u}^{3}$ & $\mathbf{u}^{2}$ & $\mathbf{u}^{2}$ & $\mathbf{u}^{2}$ & $\mathbf{u}$ & $\mathbf{u}^{1}$ & $\mathbf{u}^{\mathbf{0}}$ & $\mathbf{u}^{\mathbf{0}}$ \\
$\mathbf{1}$ & $\mathbf{2}$ & $\mathbf{3}$ & $\mathbf{3}$ & $\mathbf{5}$ & $\mathbf{3}$ & $\mathbf{3}$ & $\mathbf{6}$ & $\mathbf{2}$ & $\mathbf{2}$ & $\mathbf{3}$ & 4 \\
$\mathbf{x}_{1}$ & $\mathbf{x}_{2}$ & $\mathbf{x}_{3}$ & $\mathbf{x}_{3}$ & $\mathbf{x}_{5}$ & $\mathbf{x}_{3}$ & $\mathbf{x}_{3}$ & $\mathbf{x}_{6}$ & $\mathbf{x}_{2}$ & $\mathbf{x}_{2}$ & $\mathbf{x}_{3}$ & $\mathbf{x}_{4}$ \\
\hline
\end{tabular}

Figure 5: A fixed point when $k=3$ and $n=12$.

$a_{1}+2 a_{2}+\cdots+n a_{n}, a_{1}+\cdots+a_{n}=r_{1} \leq k$. Now suppose that $\gamma$ is the composition in a fixed point. Then if $\gamma_{i}>\gamma_{i+1}$, it cannot be that $r_{i}=r_{i+1}$ because that would violate our conditions for fixed points. Thus, it must be the case that $a_{i} \geq \chi\left(\gamma_{i}>\gamma_{i+1}\right)$. Let $x^{\gamma}$ denote $\prod_{i=1}^{n} x_{\gamma_{i}}$. In this way, the sum of the weights of all fixed points of $I$ equals

$$
\begin{aligned}
& \sum_{\gamma \in \mathbb{P}^{n}} x^{\gamma} \sum_{\substack{a_{1}+\cdots+a_{n} \leq k \\
a_{i} \geq \chi(i \in \operatorname{Des}(\gamma))}} u^{a_{1}+2 a_{2}+\cdots+n a_{n}} \\
& =\left.\sum_{\gamma \in \mathbb{P}^{n}} x^{\gamma} \sum_{a_{1} \geq \chi(1 \in \operatorname{Des}(\gamma))} \cdots \sum_{a_{n} \geq \chi(n \in \operatorname{Des}(\gamma))} y^{a_{1}+\ldots+a_{n}} u^{a_{1}+2 a_{2}+\cdots+n a_{n}}\right|_{y \leq k},
\end{aligned}
$$

where expression $\left.\right|_{t \leq k}$ means to sum the coefficients of $t^{j}$ for $j=0, \ldots, k$ in expression. Rewriting the above equation, we have

$$
\begin{aligned}
& \left.\sum_{\gamma \in \mathbb{P}^{n}} x^{\gamma} \sum_{a_{1} \geq \chi(1 \in \operatorname{Des}(\gamma))}(y u)^{a_{1}} \cdots \sum_{a_{n} \geq \chi(n \in \operatorname{Des}(\gamma))\left(y u^{n}\right)^{a_{n}}}\right|_{y \leq k} \\
& =\left.\sum_{\gamma \in \mathbb{P}^{n}} \frac{x^{\gamma}(y u)^{\chi(1 \in \operatorname{Des}(\gamma))}\left(y u^{2}\right)^{\chi(2 \in \operatorname{Des}(\gamma))} \cdots\left(y u^{n}\right)^{\chi(n \in \operatorname{Des}(\gamma))}}{(1-y u)\left(1-y u^{2}\right) \cdots\left(1-y u^{n}\right)}\right|_{y \leq k} \\
& =\left.\sum_{\gamma \in \mathbb{P}^{n}} \frac{x^{\gamma} y^{\operatorname{des}(\gamma)} u^{\operatorname{maj}(\gamma)}}{(1-y u)\left(1-y u^{2}\right) \cdots\left(1-y u^{n}\right)}\right|_{y \leq k} .
\end{aligned}
$$

Dividing by $(1-y)$ allows the above expression to be rewritten as

$$
\left.\sum_{\gamma \in \mathbb{P}^{n}} \frac{x^{\gamma} y^{\operatorname{des}(\gamma)} u^{\operatorname{maj}(\gamma)}}{(1-y)(1-y u) \cdots\left(1-y u^{n}\right)}\right|_{y^{k}} .
$$


Therefore, we have

$$
\begin{aligned}
& \sum_{n \geq 0} \frac{t^{n}}{(y ; u)_{n+1}} \sum_{\gamma \in \mathbb{P}^{n}} x^{\gamma} y^{\operatorname{des}(\gamma)} u^{\operatorname{maj}(\gamma)} \\
& =\sum_{k \geq 0} y^{k} \Theta^{(k)}\left(\sum_{n \geq 0} t^{n} h_{n}\right) \\
& =\sum_{k \geq 0} \frac{y^{k}}{\left(\sum_{n \geq 0}(-t)^{n} \Theta^{(k)}\left(e_{n}\right)\right)} \\
& =\sum_{k \geq 0} \frac{y^{k}}{\left(\left.\sum_{n \geq 0}(-t)^{n} \sum_{i_{0}, \ldots, i_{k} \geq 0} u^{0 i_{0}+\cdots+k i_{k}} \prod_{j=0}^{k} \prod_{i \geq 1}\left(1+x_{i} z_{j}\right)\right|_{z^{i} i_{0} \ldots z^{i} j}\right)} .
\end{aligned}
$$

However,

$$
\begin{aligned}
& \left.\sum_{n \geq 0}(-t)^{n} \sum_{\substack{i_{0}, \ldots, i_{k} \geq 0 \\
i_{0}+\cdots+i_{k}=n}} u^{0 i_{0}+\cdots+k i_{k}} \prod_{j=0}^{k} \prod_{i \geq 1}\left(1+x_{i} z_{j}\right)\right|_{z^{i} \ldots \ldots z^{i}} \\
& =\sum_{n \geq 0}(-t)^{n} \prod_{j=0}^{k} \prod_{i \geq 1}\left(1+u^{j} x_{i} z\right) \mid z^{n} \\
& =\prod_{j=0}^{k} \prod_{i \geq 1}\left(1-x_{i} u^{j} t\right) \\
& =\prod_{i \geq 1}\left(x_{i} t ; u\right)_{k+1} .
\end{aligned}
$$

Thus, we have shown that

$$
\sum_{n \geq 0} \frac{t^{n}}{(y ; u)_{n+1}} \sum_{\gamma \in \mathbb{P}^{n}} x^{\gamma} y^{\operatorname{des}(\gamma)} u^{\operatorname{maj}(\gamma)}=\sum_{k \geq 0} \frac{y^{k}}{\prod_{i \geq 1}\left(x_{i} t ; u\right)_{k+1}},
$$

which proves Theorem 1.1.

To prove Theorem 1.2, we define a homomorphism $\Theta_{w}^{(k)}$ on $\Lambda$ by defining

$$
\Theta_{w}^{(k)}\left(e_{n}\right)=\left.\sum_{\substack{i_{0}, \ldots, i_{k} \geq 0 \\ i_{0}+\cdots+i_{k}=n}} u^{0 i_{0}+\cdots+k i_{k}}\left[\prod_{j=0}^{k} \prod_{i \geq 1} \frac{1}{1-x_{i} z_{j}}\right]\right|_{\sum_{z_{0} \cdots} \ldots z_{k}^{i_{k}}} .
$$


Again we apply $\Theta_{w}^{(k)}$ to $h_{n}$. We have

$$
\begin{aligned}
& \Theta_{w}^{(k)}\left(h_{n}\right) \\
& =\sum_{\lambda \vdash n}(-1)^{n-\ell(\lambda)} B_{\lambda, n} \Theta_{w}^{(k)}\left(e_{\lambda}\right) \\
& =\left.\sum_{\lambda \vdash n}(-1)^{n-\ell(\lambda)} B_{\lambda, n} \prod_{m=1}^{\ell(\lambda)} \sum_{\substack{i_{0}, \ldots, i_{k} \geq 0 \\
i_{0}+\cdots+i_{k}=\lambda_{m}}} u^{0 i_{0}+\cdots+k i_{k}}\left[\prod_{j=0}^{k} \prod_{i \geq 1} \frac{1}{1-x_{i} z_{j}}\right]\right|_{z_{0}^{i_{0}} \cdots z_{k}^{i_{k}}} .
\end{aligned}
$$

Again we interpret $\Theta_{w}^{(k)}\left(h_{n}\right)$ as a sum of weighted combinatorial objects. Everything is the same as before except that the term $\left.\left[\prod_{j=0}^{k} \prod_{i \geq 1} \frac{1}{1-x_{i} z_{j}}\right]\right|_{z_{0}^{i_{0}} \ldots z_{k}^{i_{k}}}$ lets us choose $k+1$ partitions, $\pi^{(0)}, \ldots, \pi^{(k)}$ where $\ell\left(\pi^{(j)}\right)=i_{j}$ for $j=0, \ldots, k$, which we write in weakly decreasing order. Each $i$ that occurs in such a configuration is weighted with $x_{i}$, so we write these factors in the bottom row of each configuration. Figure 6 gives one example of such an object created in this manner. The weight of such a composite object is the product of the signs at the top of the configuration times the product of the $x_{i}$ 's that appear in the bottom row of the configuration times

\begin{tabular}{|c|c|c|c|c|c|c|c|c|c|c|c|}
\hline-1 & -1 & 1 & -1 & -1 & -1 & -1 & -1 & 1 & -1 & -1 & 1 \\
\hline $\mathbf{u}^{1}$ & $\mathrm{u}^{1}$ & $\mathbf{u}^{3}$ & $\mathbf{u}^{\mathbf{0}}$ & $\mathbf{u}^{0}$ & $\mathbf{u}^{0}$ & $\mathbf{u}^{2}$ & $u^{2}$ & $u^{2}$ & $\mathbf{u}^{0}$ & $\mathbf{u}^{3}$ & $u^{3}$ \\
\hline 6 & 1 & 3 & 3 & 3 & 2 & 4 & 3 & 2 & 2 & 4 & 4 \\
\hline $\mathbf{x}_{6}$ & $\mathbf{x}_{1}$ & $\mathbf{x}_{3}$ & $\mathbf{x}_{3}$ & $\mathbf{x}_{3}$ & $\mathbf{x}_{2}$ & $\mathbf{x}$ & $\mathbf{x}_{3}$ & $\mathbf{x}_{2}$ & $\mathbf{x}_{2}$ & $\mathbf{x}_{4}$ & $x_{4}$ \\
\hline
\end{tabular}
the products of the $u^{j}$ 's in the second row of the configuration. Thus, the weight of the object in Figure 6 is $-x_{1} x_{2}^{3} x_{3}^{4} x_{4}^{3} x_{6} u^{17}$.

Figure 6: An object coming from (3.2) when $k=3$ and $n=12$.

These decorated brick tabloids of shape $(n)$ and type $\lambda$ for some $\lambda \vdash n$ have the following properties:

(1) the cells in each brick contain -1 except for the final cell, which contains 1 ,

(2) each cell contains a power of $u$ such that the powers weakly increase within each brick and the largest possible power of $u$ is $u^{k}$, and

(3) $T$ contains a composition of $n$ which must weakly decrease between consecutive cells within a brick if the cells are marked with the same power of $u$.

In addition, each entry $i$ in the composition is weighted by $x_{i}$. In this way, $\Theta_{w}^{(k)}\left(h_{n}\right)$ is the weighted sum over all possible decorated brick tabloids.

We define a sign-reversing involution $I$ exactly as before. That is, we scan the cells from left to right looking for either a cell containing -1 or two consecutive bricks which may be combined to preserve the properties of this collection of objects. If $\mathrm{a}-1$ is scanned first, break the brick containing the -1 into two immediately after 
the violation and change the -1 to 1 . If the second situation is scanned first, glue the brick together and change the 1 in the first brick to -1 . Thus, $I$ shows that $\Theta_{w}^{(k)}\left(h_{n}\right)$ is equal to the sum of the weights of all the fixed points of $I$.

Again, let us consider the fixed points of $I$. First, there can be no -1 's, so every brick must be of size 1 . Next, it cannot be the case that the power of $u$ strictly increases as we move from brick $i$ to brick $i+1$, since then we could combine these two bricks and still satisfy properties (1), (2), and (3). Thus, the powers of $u$ must weakly decrease as we read from left to right. Let $\gamma=\left(\gamma_{1}, \ldots, \gamma_{n}\right)$ denote the underlying composition. We note that if the power of $u$ is the same on bricks $i$ and $i+1$, then it must be the case that $\gamma_{i}<\gamma_{i+1}$ : Otherwise, we could combine brick $i$ and brick $i+1$. One example of a fixed point may be found in Figure 7 .

\begin{tabular}{|c|c|c|c|c|c|c|c|c|c|c|c|}
\hline $\mathbf{1}$ & $\mathbf{1}$ & $\mathbf{1}$ & $\mathbf{1}$ & $\mathbf{1}$ & $\mathbf{1}$ & $\mathbf{1}$ & $\mathbf{1}$ & $\mathbf{1}$ & $\mathbf{1}$ & $\mathbf{1}$ & $\mathbf{1}$ \\
$\mathbf{u}^{3}$ & $\mathbf{u}^{3}$ & $\mathbf{u}^{3}$ & $\mathbf{u}^{3}$ & $\mathbf{u}^{3}$ & $\mathbf{u}^{2}$ & $\mathbf{u}^{2}$ & $\mathbf{u}^{2}$ & $\mathbf{u}^{1}$ & $\mathbf{u}^{1}$ & $\mathbf{u}^{0}$ & $\mathbf{u}^{0}$ \\
1 & 2 & 3 & 4 & 6 & 3 & 5 & 6 & 2 & 5 & 3 & 4 \\
$\mathbf{x}_{1}$ & $\mathbf{x}_{2}$ & $\mathbf{x}_{3}$ & $\mathbf{x}_{4}$ & $\mathbf{x}_{6}$ & $\mathbf{x}_{3}$ & $\mathbf{x}_{5}$ & $\mathbf{x}_{6}$ & $\mathbf{x}_{2}$ & $\mathbf{x}_{5}$ & $\mathbf{x}_{3}$ & $\mathbf{x}_{4}$ \\
\hline
\end{tabular}

Figure 7: A fixed point when $k=3$ and $n=12$.

We can then count the fixed points as before. That is, suppose that the powers of $u$ in a fixed point are $r_{1}, \ldots, r_{n}$ when read from left to right. It must be the case that $k \geq r_{1} \geq \cdots \geq r_{n}$. Define nonnegative integers $a_{i}$ by $a_{i}=r_{i}-r_{i+1}$ for $i=1, \ldots, n-1$ and let $a_{n}=r_{n}$. It follows that $r_{1}+\cdots+r_{n}=a_{1}+2 a_{2}+\cdots+n a_{n}, a_{1}+\cdots+a_{n}=$ $r_{1} \leq k$. Now, suppose $\gamma$ is the composition in a fixed point. Then if $\gamma_{i} \geq \gamma_{i+1}$, it cannot be that $r_{i}=r_{i+1}$ because that would violate our conditions for fixed points. Thus, it must be the case that $a_{i} \geq \chi\left(\gamma_{i} \geq \gamma_{i+1}\right)$. Let $x^{\gamma}$ denote $\prod_{i=1}^{n} x_{\gamma_{i}}$. In this way, the sum the weights of all fixed points of $I$ equals

$$
\begin{aligned}
& \sum_{\gamma \in \mathbb{P}^{n}} x^{\gamma} \sum_{\substack{a_{1}+\cdots+a_{n} \leq k \\
a_{i} \geq \chi(i \in W \operatorname{Des}(\gamma))}} u^{a_{1}+2 a_{2}+\cdots+n a_{n}} \\
& =\left.\sum_{\gamma \in \mathbb{P}^{n}} x^{\gamma} \sum_{a_{1} \geq \chi(1 \in W \operatorname{Des}(\gamma))} \cdots \sum_{a_{n} \geq \chi(n \in W \operatorname{Des}(\gamma))} y^{a_{1}+\cdots+a_{n}} u^{a_{1}+2 a_{2}+\cdots+n a_{n}}\right|_{y \leq k} .
\end{aligned}
$$

Rewriting the above equation, we have

$$
\begin{aligned}
& \left.\sum_{\gamma \in \mathbb{P}^{n}} x^{\gamma} \sum_{a_{1} \geq \chi(1 \in W \operatorname{Des}(\gamma))}(y u)^{a_{1}} \cdots \sum_{a_{n} \geq \chi(n \in W \operatorname{Des}(\gamma))}\left(y u^{n}\right)^{a_{n}}\right|_{y \leq k} \\
& =\left.\sum_{\gamma \in \mathbb{P}^{n}} \frac{x^{\gamma}(y u)^{\chi(1 \in W \operatorname{Des}(\gamma))}\left(y u^{2}\right)^{\chi(2 \in W \operatorname{Des}(\gamma))} \cdots\left(y u^{n}\right)^{\chi(n \in W D e s(\sigma))}}{(1-y u)\left(1-y u^{2}\right) \cdots\left(1-y u^{n}\right)}\right|_{y \leq k}
\end{aligned}
$$




$$
=\left.\sum_{\gamma \in \mathbb{P}^{n}} \frac{x^{\gamma} y^{\operatorname{wdes}(\gamma)} u^{\operatorname{wmaj}(\gamma)}}{(1-y u)\left(1-y u^{2}\right) \cdots\left(1-y u^{n}\right)}\right|_{y \leq k} .
$$

Dividing by $(1-y)$ allows the above expression to be rewritten as

$$
\left.\sum_{\gamma \in \mathbb{P}^{n}} \frac{x^{\gamma} y^{\operatorname{wdes}(\gamma)} u^{\operatorname{wmaj}(\gamma)}}{(1-y)(1-y u) \cdots\left(1-y u^{n}\right)}\right|_{y^{k}} .
$$

Therefore, we have

$$
\begin{aligned}
& \sum_{n \geq 0} \frac{t^{n}}{(y ; u)_{n+1}} \sum_{\gamma \in \mathbb{P}^{n}} x^{\gamma} y^{\mathrm{wdes}(\gamma)} q^{\mathrm{wmaj}(\gamma)} \\
& =\sum_{k \geq 0} y^{k} \Theta_{w}^{(k)}\left(\sum_{n \geq 0} t^{n} h_{n}\right) \\
& =\sum_{k \geq 0} \frac{y^{k}}{\left(\sum_{n \geq 0}(-t)^{n} \Theta_{w}^{(k)}\left(e_{n}\right)\right)} \\
& =\sum_{k \geq 0} \frac{y^{k}}{\left(\left.\sum_{n \geq 0}(-t)^{n} \sum_{i_{0}, \ldots, i_{k} \geq 0} u^{0 i_{0}+\cdots+k i_{k}} \prod_{j=0}^{k} \prod_{i \geq 1} \frac{1}{1-x_{i} z_{j}}\right|_{z_{0}^{i_{0}} \ldots z_{k}} ^{i_{k}}\right)} .
\end{aligned}
$$

However,

$$
\begin{aligned}
\left.\sum_{n \geq 0}(-t)^{n} \sum_{\substack{i_{0}, \ldots, i_{k} \geq 0 \\
i_{0}+\cdots+i_{k}=n}} u^{0 i_{0}+\cdots+k i_{k}} \prod_{j=0}^{k} \prod_{i \geq 1} \frac{1}{1-x_{i} z_{j}}\right|_{z_{0} i_{0} \ldots z_{k}^{i_{k}}} & =\sum_{n \geq 0}(-t)^{n} \prod_{j=0}^{k} \prod_{i \geq 1} \frac{1}{1-u^{j} x_{i} z} \mid z^{n} \\
& =\prod_{j=0}^{k} \prod_{i \geq 1} \frac{1}{1+x_{i} u^{j} t} \\
& =\prod_{i \geq 1} \frac{1}{\left(-x_{i} t ; u\right)_{k+1}}
\end{aligned}
$$

Thus, we have shown that

$$
\sum_{n \geq 0} \frac{t^{n}}{(y ; n)_{n+1}} \sum_{\gamma \in \mathbb{P} n} x^{\gamma} y^{\mathrm{wdes}(\gamma)} u^{\mathrm{wmaj}(\gamma)}=\sum_{k \geq 0} y^{k} \prod_{i \geq 1}\left(-x_{i} t ; u\right)_{k+1},
$$

which proves Theorem 1.2.

To prove Theorem 1.3, we define a homomorphism $\Theta_{\ell}^{(k)}\left(e_{n}\right)$ on $\Lambda$ by setting

$$
\Theta_{\ell}^{(k)}\left(e_{n}\right)=\sum_{\substack{i_{0}, \ldots, i_{k} \geq 0 \\ i_{0}+\cdots+i_{k}=n}} u^{0 i_{0}+\cdots+k i_{k}} \prod_{j=0}^{k} p_{i_{j}}
$$


where $p_{n}$ is the $n$-th power symmetric function.

As before, apply $\Theta_{\ell}^{(k)}$ to $h_{n}$ we have

$$
\begin{aligned}
\Theta_{\ell}^{(k)}\left(h_{n}\right) & =\sum_{\lambda \vdash n}(-1)^{n-\ell(\lambda)} B_{\lambda, n} \Theta^{(k)}\left(e_{\lambda}\right) \\
& =\sum_{\lambda \vdash n}(-1)^{n-\ell(\lambda)} B_{\lambda, n} \prod_{m=1}^{\ell(\lambda)} \sum_{\substack{i_{0}, \ldots, i_{k} \geq 0 \\
i_{0}+\cdots+i_{k}=\lambda_{m}}} u^{0 i_{0}+\cdots+k i_{k}} p_{i_{0}} \cdots p_{i_{k}} .
\end{aligned}
$$

Again we interpret $\Theta_{\ell}^{(k)}\left(h_{n}\right)$ as a sum of weighted combinatorial objects. Everything is the same as before except that the term $p_{i_{0}} \cdots p_{i_{k}}$ lets us choose $k+1$ partitions, $\pi^{(0)}, \ldots, \pi^{(k)}$ where $\pi^{(j)}=\left(n_{j}^{i_{j}}\right)$ for some $n_{j}$ for $j=0, \ldots, k$. Each $i$ that occurs in such a configuration is weighted with $x_{i}$ so that we write these factors in the bottom row of each configuration. Figure 8 gives one example of such an object created in this manner. The weight of such a composite object is the product of the signs at the top of the configuration times the product of the $x_{i}$ 's that appear in the bottom row of the configuration times the products of the $u^{j}$ 's in the second row of

\begin{tabular}{|c|c|c|c|c|c|c|c|c|c|c|c|}
\hline-1 & -1 & 1 & -1 & -1 & -1 & -1 & -1 & 1 & -1 & -1 & 1 \\
\hline$u^{1}$ & $u^{1}$ & $u^{3}$ & $\mathbf{u}^{0}$ & $\mathbf{u}^{0}$ & $\mathbf{u}^{0}$ & $u^{2}$ & $u^{2}$ & $u^{2}$ & $\mathbf{u}^{\mathbf{0}}$ & $u^{3}$ & $\mathbf{u}^{3}$ \\
\hline 6 & 6 & 3 & 3 & 3 & 3 & 4 & 4 & 4 & 2 & 3 & 3 \\
\hline $\mathbf{x}_{6}$ & $\mathbf{x}_{6}$ & $\mathbf{x}_{3}$ & $\mathbf{x}_{3}$ & $\mathbf{x}_{3}$ & $\mathbf{x}_{3}$ & $\mathbf{x}_{4}$ & $\mathbf{x}_{4}$ & $x_{4}$ & $\mathbf{x}_{2}$ & $\mathbf{x}_{\mathbf{3}}$ & $\mathbf{x}_{3}$ \\
\hline
\end{tabular}
the configuration. Thus, the weight of the object in Figure 8 is $-x_{2} x_{3}^{6} x_{4}^{3} x_{6}^{2} u^{17}$.

Figure 8: An object coming from (3.3) when $k=3$ and $n=12$.

These decorated brick tabloids of shape $(n)$ and type $\lambda$ for some $\lambda \vdash n$ have the following properties:

(1) the cells in each brick contain -1 except for the final cell, which contains 1 ,

(2) each cell contains a power of $u$ such that the powers weakly increase within each brick and the largest possible power of $u$ is $u^{k}$, and

(3) $T$ contains a composition of $n$ whose entries must be equal for any two consecutive cells within a brick if the cells are marked with the same power of $u$.

In addition, each entry $i$ in the composition is weighted by $x_{i}$. In this way, $\Theta_{\ell}^{(k)}\left(h_{n}\right)$ is the weighted sum over all possible decorated brick tabloids.

We define a sign-reversing involution $I$ exactly as before. That is, we scan the cells from left to right looking for either a cell containing -1 or two consecutive bricks which may be combined to preserve the properties of this collection of objects. If $a-1$ is scanned first, break the brick containing the -1 into two immediately after the violation and change the -1 to 1 . If the second situation is scanned first, glue the 
brick together and change the 1 in the first brick to -1 . Thus, $I$ shows that $\Theta_{\ell}^{(k)}\left(h_{n}\right)$ is equal to the sum of the weights of all the fixed points of $I$.

Again, let us consider the fixed points of $I$. First, there can be no -1 's, so every brick must be of size 1 . Next, it cannot be the case that the power of $u$ strictly increases as we move from brick $i$ to brick $i+1$, since then we could combine these two bricks and still satisfy properties (1), (2), and (3). Thus, the powers of $u$ must weakly decrease as we read from left to right. Let $\gamma=\left(\gamma_{1}, \ldots, \gamma_{n}\right)$ denote the underlying composition. We note that if the power of $u$ is the same on brick $i$ and $i+1$, then it must be the case that $\gamma_{i} \neq \gamma_{i+1}$; otherwise, we could combine brick $i$ and brick $i+1$. One example of a fixed point may be found in Figure 9.

\begin{tabular}{|c|c|c|c|c|c|c|c|c|c|c|c|}
\hline $\begin{array}{c}1 \\
\mathbf{u}^{3}\end{array}$ & $\mathbf{u}^{\mathbf{3}}$ & $\begin{array}{c}\mathbf{1} \\
u^{3}\end{array}$ & $\begin{array}{c}\mathbf{1}^{3} \\
\mathbf{u}^{3}\end{array}$ & $\begin{array}{c}1 \\
u^{3}\end{array}$ & $\begin{array}{c}1 \\
\mathbf{u}^{2}\end{array}$ & $\begin{array}{c}1 \\
\mathbf{u}^{2}\end{array}$ & $\begin{array}{c}\mathbf{1}^{2} \\
\mathbf{u}^{2}\end{array}$ & $\begin{array}{c}1 \\
u^{1}\end{array}$ & $\begin{array}{c}1 \\
u^{1}\end{array}$ & $\begin{array}{c}\mathbf{u}^{0} \\
\end{array}$ & $\begin{array}{c}\mathbf{1} \\
\mathbf{u}^{0}\end{array}$ \\
\hline 3 & 2 & 1 & 3 & 5 & 3 & 2 & 6 & 6 & 2 & 3 & 4 \\
\hline $\mathbf{x}_{3}$ & $\mathbf{x}_{2}$ & $\mathbf{x}_{1}$ & $\mathbf{x}_{3}$ & $\mathbf{x}_{5}$ & $\mathbf{x}_{3}$ & $\mathbf{x}_{2}$ & $\mathbf{x}_{6}$ & $\mathbf{x}_{6}$ & $\mathbf{x}_{2}$ & $\mathbf{x}_{3}$ & $\mathbf{x}_{4}$ \\
\hline
\end{tabular}

Figure 9: A fixed point when $k=3$ and $n=12$.

We can then count the fixed points as before. That is, suppose that the powers of $u$ in a fixed point are $r_{1}, \ldots, r_{n}$ when read from left to right. It must be the case that $k \geq r_{1} \geq \cdots \geq r_{n}$. Define nonnegative integers $a_{i}$ by $a_{i}=r_{i}-r_{i+1}$ for $i=1, \ldots, n-1$ and let $a_{n}=r_{n}$. It follows that $r_{1}+\cdots+r_{n}=a_{1}+2 a_{2}+\cdots+n a_{n}, a_{1}+\cdots+a_{n}=$ $r_{1} \leq k$. Now suppose that $\gamma$ is the composition in a fixed point. Then if $\gamma_{i}=\gamma_{i+1}$, then it cannot be that $r_{i}=r_{i+1}$ because that would violate our conditions for fixed points. Thus, it must be the case that $a_{i} \geq \chi\left(\gamma_{i}=\gamma_{i+1}\right)$. Let $x^{\gamma}$ denote $\prod_{i=1}^{n} x_{\gamma_{i}}$. In this way, the sum of the weights of all fixed points of $I$ equals

$$
\begin{aligned}
& \sum_{\gamma \in \mathbb{P}^{n}} x^{\gamma} \sum_{\substack{a_{1}+\cdots+a_{n} \leq k \\
a_{i} \geq \chi(i \in \operatorname{Lev}(\gamma))}} u^{a_{1}+2 a_{2}+\cdots+n a_{n}} \\
& =\left.\sum_{\gamma \in \mathbb{P}^{n}} x^{\gamma} \sum_{a_{1} \geq \chi(1 \in \operatorname{Lev}(\gamma))} \cdots \sum_{a_{n} \geq \chi(n \in \operatorname{Lev}(\gamma))} y^{a_{1}+\cdots+a_{n}} u^{a_{1}+2 a_{2}+\cdots+n a_{n}}\right|_{y \leq k} .
\end{aligned}
$$

Rewriting the above equation, we have

$$
\begin{aligned}
& \left.\sum_{\gamma \in \mathbb{P}^{n}} x^{\gamma} \sum_{a_{1} \geq \chi(1 \in \operatorname{Lev}(\gamma))}(y u)^{a_{1}} \cdots \sum_{a_{n} \geq \chi(n \in \operatorname{Lev}(\gamma))}\left(y u^{n}\right)^{a_{n}}\right|_{y \leq k} \\
& =\left.\sum_{\gamma \in \mathbb{P}^{n}} \frac{x^{\gamma}(y u)^{\chi(1 \in \operatorname{Lev}(\gamma))}\left(y u^{2}\right)^{\chi(2 \in \operatorname{Lev}(\gamma))} \cdots\left(y u^{n}\right)^{\chi(n \in \operatorname{Lev}(\gamma))}}{(1-y u)\left(1-y u^{2}\right) \cdots\left(1-y u^{n}\right)}\right|_{y \leq k} \\
& =\left.\sum_{\gamma \in \mathbb{P}^{n}} \frac{x^{\gamma} y^{\operatorname{lev}(\gamma)} u^{\operatorname{levmaj}(\gamma)}}{(1-y u)\left(1-y u^{2}\right) \cdots\left(1-y u^{n}\right)}\right|_{y \leq k} .
\end{aligned}
$$


Dividing by $(1-y)$ allows the above expression to be rewritten as

$$
\left.\sum_{\gamma \in \mathbb{P}^{n}} \frac{x^{\gamma} y^{\operatorname{lev}(\gamma)} u^{\operatorname{levmaj}(\gamma)}}{(1-y)(1-y u) \cdots\left(1-y u^{n}\right)}\right|_{y^{k}} .
$$

Therefore, we have

$$
\begin{aligned}
& \sum_{n \geq 0} \frac{t^{n}}{(y ; u)_{n+1}} \sum_{\gamma \in \mathbb{P}^{n}} x^{\gamma} y^{\operatorname{lev}(\gamma)} u^{\operatorname{levmaj}(\gamma)} \\
& =\sum_{k \geq 0} y^{k} \Theta_{\ell}^{(k)}\left(\sum_{n \geq 0} t^{n} h_{n}\right) \\
& =\sum_{k \geq 0} \frac{y^{k}}{\left(\sum_{n \geq 0}(-t)^{n} \Theta_{\ell}^{(k)}\left(e_{n}\right)\right)} \\
& \left.=\sum_{k \geq 0} \frac{y^{k}}{\left(\sum_{n \geq 0}(-t)^{n} \sum_{\substack{i_{0}, \ldots, i_{k} \geq 0 \\
i_{0}+\cdots+i_{k}=n}} u^{0 i_{0}+\cdots+k i_{k}} p_{i_{0}} \cdots p_{i_{j}}\right.}\right) .
\end{aligned}
$$

However,

$$
\begin{aligned}
\sum_{n \geq 0}(-t)^{n} \sum_{\substack{i_{0}, \ldots, i_{k} \geq 0 \\
i_{0}+\cdots+i_{k}=n}} u^{0 i_{0}+\cdots+k i_{k}} p_{i_{0}} \cdots p_{i_{j}} & =\sum_{n \geq 0}(-t)^{n} \prod_{j=0}^{k}\left(\sum_{m \geq 0} p_{m}\left(u^{j} z\right)^{m}\right) \mid z^{n} \\
& =\prod_{j=0}^{k}\left(\sum_{m \geq 0} p_{m}\left(-u^{j} t\right)^{m}\right)
\end{aligned}
$$

Thus, we have shown that

$$
\sum_{n \geq 0} \frac{t^{n}}{(y ; u)_{n+1}} \sum_{\gamma \in \mathbb{P}^{n}} x^{\gamma} y^{\operatorname{lev}(\gamma)} u^{\operatorname{levmaj}(\gamma)}=\sum_{k \geq 0} \frac{y^{k}}{\prod_{j=0}^{k}\left(\sum_{0 \geq 1} p_{m}\left(-u^{j} t\right)^{m}\right)},
$$

which proves Theorem 1.3.

Now suppose that $S$ is a subset of $\mathbb{P}$. Then we can restrict to compositions with parts from $S$ by simply setting $x_{i}=0$ for all $i \notin S$. Thus, we immediately have the following three corollaries:

Corollary 3.1.

$$
\sum_{n \geq 0} \frac{t^{n}}{(y ; u)_{n+1}} \sum_{\gamma \in S^{n}} x^{\gamma} y^{\operatorname{des}(\gamma)} u^{\operatorname{maj}(\gamma)}=\sum_{k \geq 0} \frac{y^{k}}{\prod_{i \in S}\left(x_{i} t ; u\right)_{k+1}}
$$

\section{Corollary 3.2.}

$$
\sum_{n \geq 0} \frac{t^{n}}{(y ; u)_{n+1}} \sum_{\gamma \in S^{n}} x^{\gamma} y^{\operatorname{wdes}(\gamma)} u^{\operatorname{wmaj}(\gamma)}=\sum_{k \geq 0} y^{k} \prod_{i \in S}\left(-x_{i} t ; u\right)_{k+1} .
$$




\section{Corollary 3.3.}

$$
\sum_{n \geq 0} \frac{t^{n}}{(y ; u)_{n+1}} \sum_{\gamma \in S^{n}} x^{\gamma} y^{\operatorname{lev}(\gamma)} u^{\operatorname{levmaj}(\gamma)}=\sum_{k \geq 0} \frac{y^{k}}{\prod_{j=0}^{k}\left(\sum_{n \geq 0} p_{n, S}\left(-u^{j} t\right)^{n}\right)},
$$

where $p_{n, S}=\sum_{i \in S} x_{i}^{n}$.

We can also derive analogues of our results for other partial orders on $\mathbb{P}$ by specializing our results. For instance, suppose that $\preccurlyeq$ is the partial order where all the odd numbers are incomparable, every even number is larger than every odd number, and the even numbers are ordered as in the standard universe. In this case, we define for any composition $\gamma=\left(\gamma_{1}, \ldots, \gamma_{n}\right)$,

$$
\begin{aligned}
& \overline{\operatorname{Des}}(\gamma)=\left\{i: \gamma_{i} \succ \gamma_{i+1}\right\}, \\
& \overline{\operatorname{des}}(\gamma)=|\overline{\operatorname{Des}}(\gamma)|, \text { and } \\
& \overline{\operatorname{maj}}(\gamma)=\sum_{i \in \overline{\operatorname{Des}}(\gamma)} i .
\end{aligned}
$$

Then it is easy to see that the generating function

$$
\sum_{n \geq 0} \frac{t^{n}}{(y ; u)_{n+1}} \sum_{\gamma \in \mathbb{P} n} x^{\gamma} y^{\overline{\operatorname{des}}(\gamma)} u^{\overline{\operatorname{maj}}(\gamma)}
$$

arises by taking the generating function

$$
\sum_{n \geq 0} \frac{t^{n}}{(y ; u)_{n+1}} \sum_{\gamma \in \mathbb{P}^{n}} x^{\gamma} y^{\operatorname{des}(\gamma)} u^{\operatorname{maj}(\gamma)}
$$

and setting $x_{1}=\frac{1}{1-\sum_{n \geq 0} x_{2 n+1}}$ and setting $x_{2 i+1}=0$ for $i \geq 1$. Thus, we have the following corollary.

\section{Corollary 3.4.}

$$
\sum_{n \geq 0} \frac{t^{n}}{(y ; u)_{n+1}} \sum_{\gamma \in \mathbb{P}^{n}} x^{\gamma} y^{\overline{\operatorname{des}}(\gamma)} u^{\overline{\operatorname{maj}}(\gamma)}=\sum_{k \geq 0} \frac{y^{k}}{\prod_{j=0}^{k}\left(1-\frac{u^{j} t}{1-\sum_{n \geq 0} x_{2 n+1}}\right) \prod_{i \geq 1}\left(x_{2 i} t ; u\right)_{k+1}}
$$

\section{References}

1. Andrews, G.: The Theory of Partitions. Cambridge University Press, Cambridge (1998)

2. Beck, D.: Permutation enumeration of the symmetric and hyperoctahedral group and the combinatorics of symmetric functions. Ph.D. Thesis, University of California, San Diego (1993)

3. Beck, D., Remmel, J.: Permutation enumeration of the symmetric group and the combinatorics of symmetric functions, J. Combin. Theory Ser. A 72(1), 1-49 (1995)

4. Beck, D.: The combinatorics of symmetric functions and permutation enumeration of the hyperoctahedral group. Discrete Math. 163(1-3), 13-45 (1997) 
5. Brenti, F.: Permutation enumeration, symmetric functions, and unimodality. Pacific J. Math. 157(1), 1-28 (1993)

6. Brenti, F.: Unimodal polynomials arising from symmetric functions. Proc. Amer. Math. Soc. 108(4), 1133-1141 (1990)

7. Carlitz, L.: Enumeration of compositions by rises, falls, and levels. Math. Nachr. 77(3), 254-264 (1977)

8. Eğecioğlu, Ö., Remmel, J.: Brick tabloids and the connection matrices between bases of symmetric functions. Discrete Appl. Math. 34(1-3), 107-120 (1991)

9. Garsia, A., Gessel, I.: Permutation statistics and partitions. Adv. Math. 31(3), 288-305 (1979)

10. Gessel, I.: Generating functions and enumeration of sequences. Ph.D. Thesis, MIT, Cambridge (1977)

11. Goulden, I.P., Jackson, D.M.: Combinatorial Enumeration. John Wiley \& Sons, New York (1983)

12. Heubach, S., Mansour, T.: Counting rises, levels, and drops in compositions. Integers 5(1), \#A11 (2005)

13. Heubach, S., Mansour, T.: Enumeration of 3-letter patterns in compositions. In: Combinatorial Number Theory, pp. 243-264. de Gruyter, Berlin (2007)

14. Heubach, S., Kitaev, S., Mansour, T.: Partially ordered patterns and compositions. Pure Math. Appl. 17(1-2), 123-134 (2006)

15. Kitaev, S., Mansour, T., Remmel, J.: Counting descents, rises, and levels with prescribed first elements in words. Discrete Math. Theor. Comput. Sci. 10(3), 1-22 (2008)

16. MacMahon, P.: Combinatory Analysis, Vols. 1 and 2. Cambridge University Press, Cambridge (1915)

17. Mansour, T., Sirhan, B.: Counting l-letter subwords in compositions. Discrete Math. Theor. Comput. Sci. 8(1), 285-297 (2006)

18. Mendes, A., Remmel, J.: Descents, inversions, and major indices in permutation groups. Discrete Math. 308(12), 2509-2524 (2007)

19. Reiner, V.: Signed permutation statistics. European J. Combin. 14(6), 553-567 (1993)

20. Stanley, R.: Enumerative Combinatorics, Vol. 1. Cambridge University Press, Cambridge (1997)

Open Access This article is distributed under the terms of the Creative Commons Attribution Noncommercial License which permits any noncommercial use, distribution, and reproduction in any medium, provided the original author(s) and source are credited. 